\title{
DESIGNING A WEB APPLICATION USING AN ETHNOGRAPHIC RESEARCH APPROACH
}

\author{
Nanda C. Surendra \\ email: nsurendra@pace.edu
}

\begin{abstract}
In this research-in-progress, I am working with users in collaboratively designing and developing a web application that can serve as a support tool for stock analysis. I am using an ethnographic research approach called the Strip Resolution process (Agar, 1986) as a research tool to better explain the process by which I develop and validate an interpretation as a researcher. When using the Strip Resolution process as a research tool, I found that it could also be used as a means of understanding users' requirements to better design the application.
\end{abstract}

I begin this paper by introducing the questions that motivate my research and the research approach I have chosen as being suitable to study those questions. One element of my research approach is the Strip Resolution process. In the second section, I discuss what constitutes this process. In the third section, I trace how this ethnographic approach enabled me to better understand one of the major work processes (called "evaluating stock holding") of stock analysts, who are the primary end users for this application. This understanding of the stock analysts' work process improved my understanding of their requirements - thereby, enabling better design of the application to meet their needs. In the final section, I compare the use of the Strip Resolution process for developing a web application with other approaches such as Extreme Programming (Beck, 2000), Adaptive Software Development (Cockburn, 2001; Highsmith, 2000), and UML-based web application development (Conallen, 2000; Rosenberg and Scott, 2001).

Key words: Web application development, Ethnography

The original version of this chapter was revised: The copyright line was incorrect. This has been corrected. The Erratum to this chapter is available at DOI: 10.1007/978-0-387-35604-4_20 


\section{MY RESEARCH OBJECTIVES AND RESEARCH APPROACH}

Two questions of practical relevance motivate my research. The first question is "What are the important questions to be asked as a web application designer / developer to design/develop a 'useful/ effective' system?" The second question is "What are the major problems of designing and developing a web application?"

I am exploring these npen-ended research questions in a learning mode rather than a predictive mode. In addition, my objective is to try to answer these questions in a manner that would be relevant to practitioners, even if the answers turn out to be incomplete. Hence, I am using three data collection techniques (active participation, observation, and interviews) that are a combination of ethnographic and action research approaches. I am adopting an action research perspective (Baskerville, 1999; Whyte, 1989; Whyte, Greenwood, and Lazes, 1989) by being an active participant in the roles of an application designer, an application developer, and a stock analyst (a stock analyst is the primary end-user of the application). I am adopting an ethnographic perspective (Agar, 1986) by observing the work and decision flow of stock analysts and by conducting interviews with users at all levels of the organizational hierarchy. Among the users I am interviewing are (a) Stock analysts who are the primary end-users of the application, (b) Managers of stock Analysts who are secondary end-users of the application, and (c) the head of the department in which the application will be implemented, who is the primary managerial sponsor of the project. I initially used the Strip Resolution process (Agar, 1986) as a tool in my role as a researcher to better understand and interpret the data I was collecting using the above techniques. I explain the Strip Resolution process in the next section. The potential use of this process for systems analysts and designers as a means of determining users' requirements emerged from the research process, which I explain in the third section.

\section{THE STRIP RESOLUTION PROCESS}

Agar (1986) introduced a relatively formal process of developing and validating an interpretive understanding. This process involves three notions - the breakdown, resolution, and coherence of a schema. All three notions use the "medium" of a strip to function.

A strip is data that is produced from a researcher's observations, discussion with subjects, interviews of the subjects, participation in an activity with the subjects, or study of documents. "A strip is any bounded 
phenomenon" against which an interpretive researcher "tests his or her understanding" (Agar, 1986; p.28). A schema refers to the frames of reference a researcher uses in understanding a strip. A schema breaks down when a researcher applies the schema to a strip and finds the strip not understandable. A resolution of a breakdown is the process by which the existing "broken down" schema is changed to develop a new schema that results in the strip under study being understood. Several intermediate schemas may be developed during this process of resolution before a final schema that resolves the breakdown emerges.

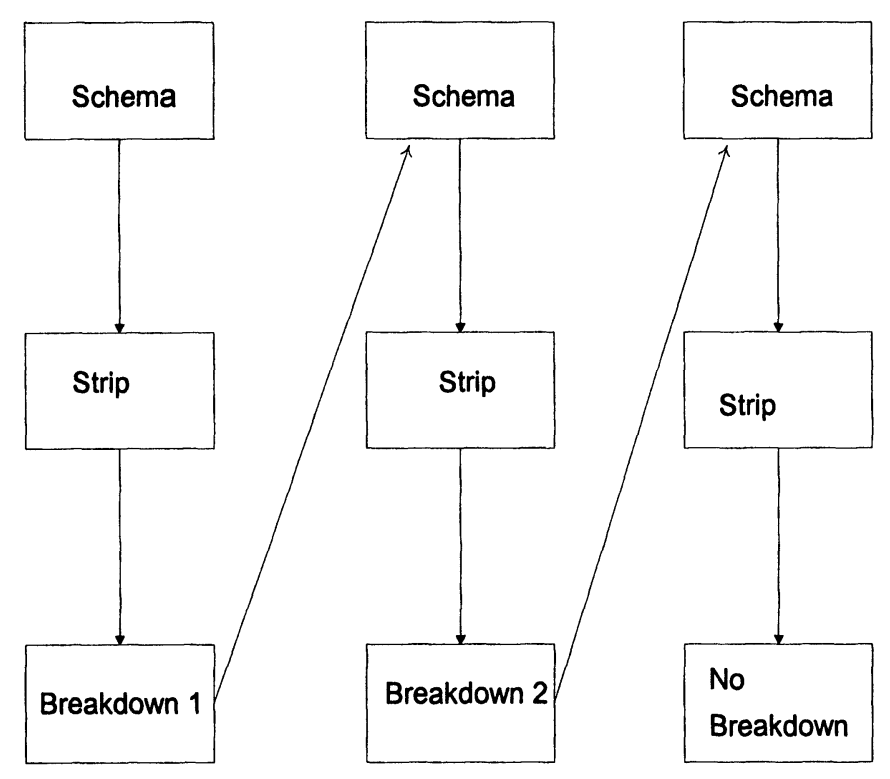

Fioure 1: The Strin Resolution

(adapted from Agar, 1986)

The new schema that enables the researcher to understand a strip is called a coherent schema. The robustness of the coherence of a schema can be "tested" by applying it to new strips. If a schema breaks down on being applied to new strips, the coherence of the schema is not strong or robust. The researcher needs to keep applying schemas to known strips until a schema that enables her to understand all strips presented is found. Such a schema is considered a strongly or robustly coherent schema. The process of resolving breakdowns and testing the coherence of schemas is the means by which the strip resolution process develops and validates an interpretive understanding.

Figure 1 illustrates the Strip Resolution process. Schema 1, the schema currently used by the researcher, is applied to a strip. The strip is not 
understood leading to Breakdown 1. Schema 1 is modified to Schema 2 and applied on the same strip. The lack of understanding of the strip continues leading to Breakdown 2. Schema 2 is changed to Schema 3 and applied to the strip, leading to an understanding of the strip. Since there is no breakdown, Schema 3 is accepted as a coherent schema. Of course, Schema 3 will have to be tested on other strips to determine if it is a "robustly" coherent schema.

\section{APPLYING THE STRIP RESOLUTION PROCESS TO UNDERSTAND HOW ANALYSTS EVALUATE STOCK HOLDINGS}

Among the major functions performed by a stock analyst are evaluating (a) how much an investment management company has invested in the stock of his client (his client is a company that has a listing on a stock exchange) - called stock holding - and, (b) whether the investment manager has been buying or selling stock belonging to his client. Figure 2 shows the path I traveled using the Strip Resolution process in developing an understanding of how stock analysts evaluated stock holdings. In the following sub-sections, I elaborate on how my understanding of "evaluating stock holdings" progressed through each step of the strip resolution process.

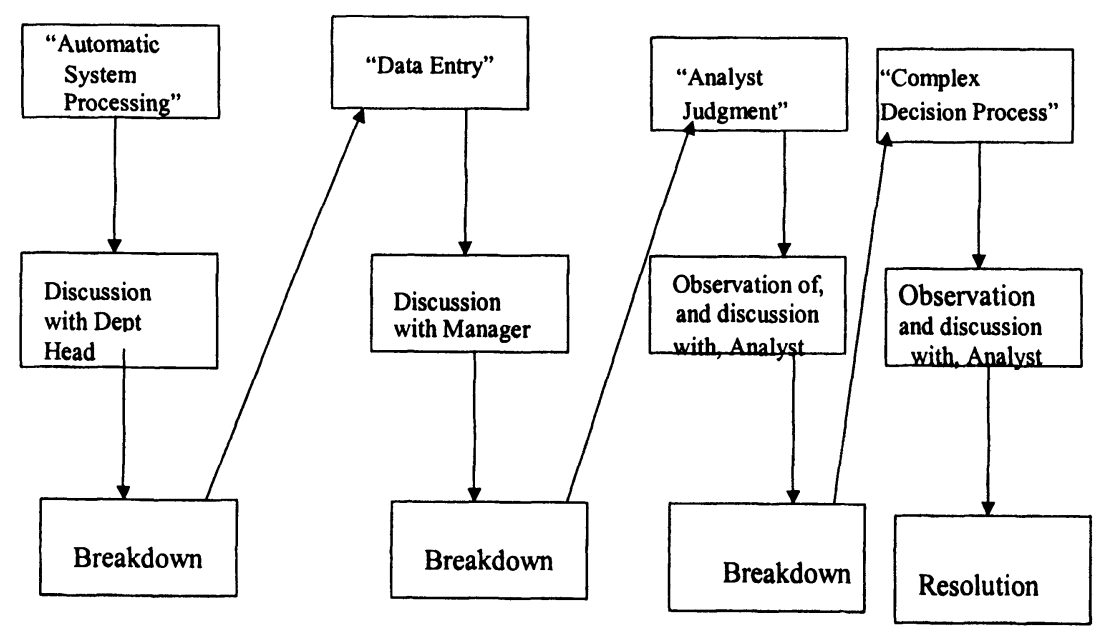

Figure 2: Applying the Strip Resolution Process:

How do analysts evaluate stock holdings? 


\subsection{Schema 1: Stock Holdings Can Be Evaluated 'Automatically' By The System}

After my initial interviews with the head of the department and one of the managers, my first schema was "Stock holdings could be evaluated 'automatically' by the system." Based on this schema, I developed a stored procedure on the database to calculate the stock holdings. When I

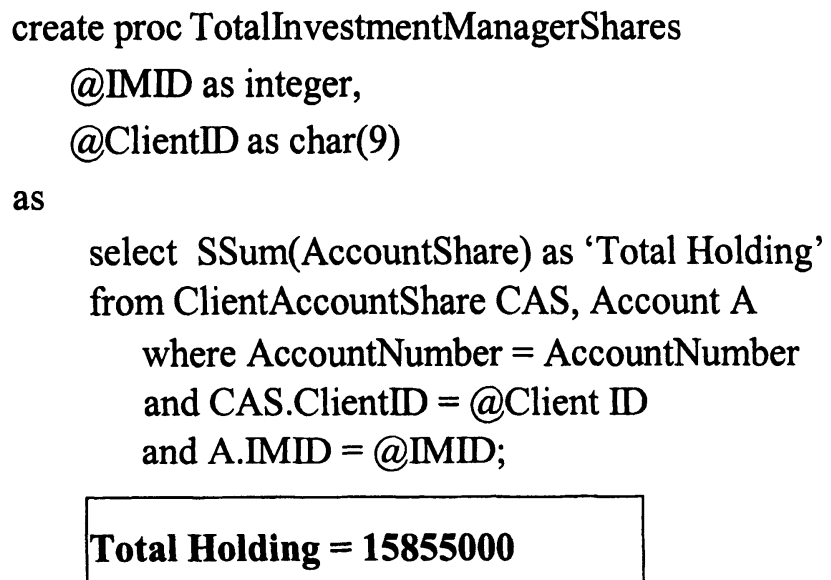

Figure 3: Schema 1

Evaluating Stock Holding as "Automatic System Processing"

demonstrated how the system calculated stock holdings to the department head, he stated his unhappiness with that approach. He said that "automatic processing" did not represent how the stock holding needed to be evaluated. So, my schema (Schema 1), on being applied to a strip represented by this discussion with the department head, broke down (Figure 4). 


\subsection{Schema 2: Evaluating Holdings Is "Data Entry"}

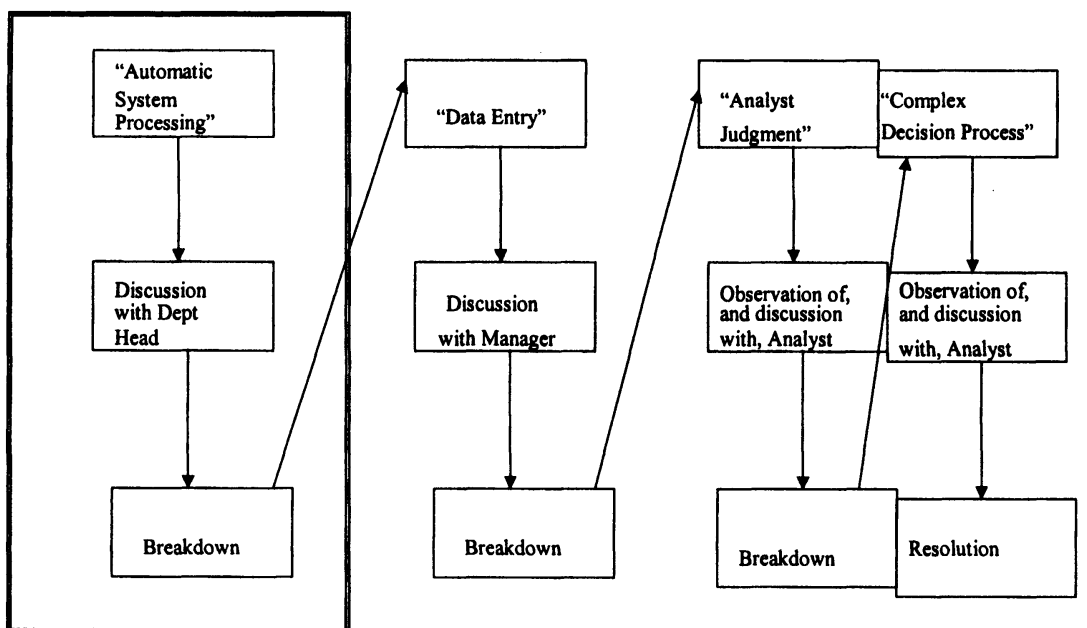

Figure 4: Breakdown of Schema 1:

Evaluate Holdings As "Automatic System Processing"

The department head emphasized that an analyst entered an estimate for stock holdings based on the values displayed for four factors (listed in Figure 5). So, my revised schema (Schema 2) was "Evaluating Holdings is a Data Entry process." Based on this schema, Schema 2, I created a tabular structure for data entry (Figure 5). While my Schema 2 resolved the breakdown on the first strip (discussion with the department head), this schema must be able to help interpret multiple strips to be considered a robust schema. My discussion with the next lower-level manager (the second strip) did not make this schema (Schema 2) robust, since he said that considering an analyst a data entry person was incorrect. So, my Schema 2 broke down on being applied to this new strip of my discussion with a manager (Figure 6).

\begin{tabular}{|l|l|}
\hline Aggregate Shares & 15850000 \\
\hline From Schedule 13 F & 17000000 \\
\hline Last Holding & 14000000 \\
\hline Investment Manager Contact & 16500000 \\
\hline Your Entry & \\
\hline Submit & \\
\hline
\end{tabular}

Figure 5: Schema 2 - Evaluate Holding as "Data Entry" 


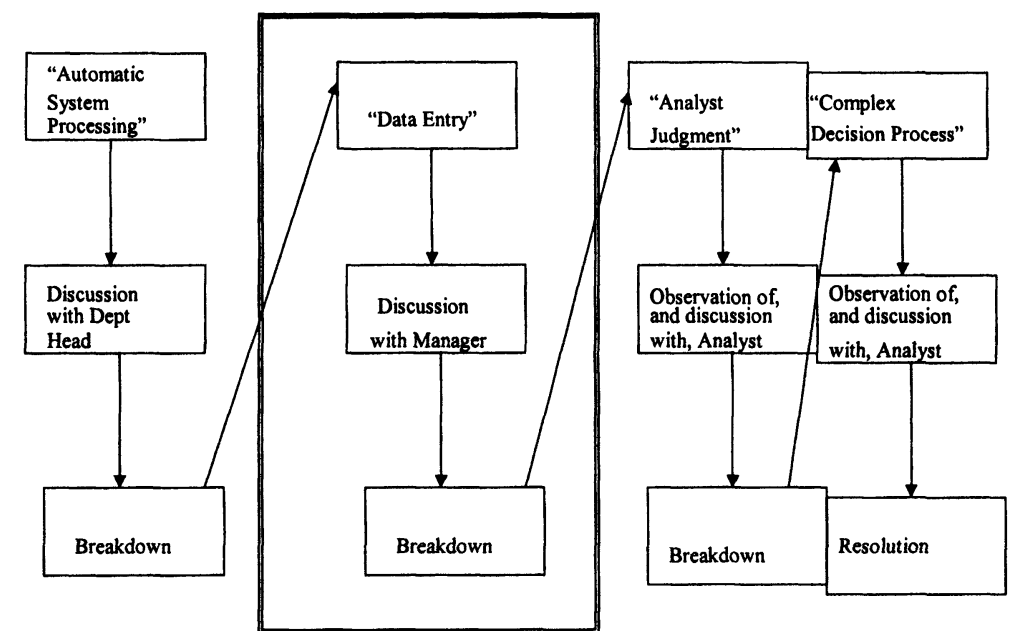

Figure 6: Breakdown of Schema 2:

Evaluate Holdings As "Data Entry"

\title{
3.3 Schema 3: Evaluating Holdings Requires "Analyst's Judgment"
}

\author{
Evaluate Holdings \\ Analyst: Hayes \\ it: Meus Wearhouse, Inc. \\ w: Crainer, Rosenthal \& McGlyum, Inc.
}

\begin{tabular}{|l|l|}
\hline Account Aggregration & 15855000 \\
\hline From Schedule 13F & 13500000 \\
\hline $\begin{array}{l}\text { Last Holding: } \\
\text { Holding Date: } \\
\text { Confidence Level } \\
\text { Comments: }\end{array}$ & 14000000 \\
$05 / 01 / 2001$ \\
\hline $\begin{array}{l}\text { Investment Manager Contact: } \\
\text { Position: } \\
\text { Telephone Number: }\end{array}$ & Account Aggregat \\
\hline Your Judgement: & $\begin{array}{l}\text { Manager } \\
\text { Smith }\end{array}$ \\
\hline Confidence Level: & \\
\hline Notes: & \\
\hline \multicolumn{1}{|c|}{ Submit New Holding } \\
\hline
\end{tabular}

Figure 7: Schema 3: Evaluate Holdings as "Analyst Judgment" 
My discussion with this second tier manager led to a revision of my schema - my re-revised schema (Schema 3) was that "Evaluating Holdings required an analyst's judgment." I represented my new schema on the prototype (shown in Figure 7) by providing a confidence level indicator to the estimate entered by the analyst and by providing an area for notes to enable the analyst to record the factors that led to the analyst's judgment. However, Schema 3 also did not prove to be robust - it broke down when I was observing the workflow / decision flow of the analysts (who were referring to many more factors than were represented on prototype in Figure 7) and when I requested an analyst to work with the prototype (these observations constituted the third strip). Analysts considered other factors in addition to the four represented in Figure 7 before making their judgment.

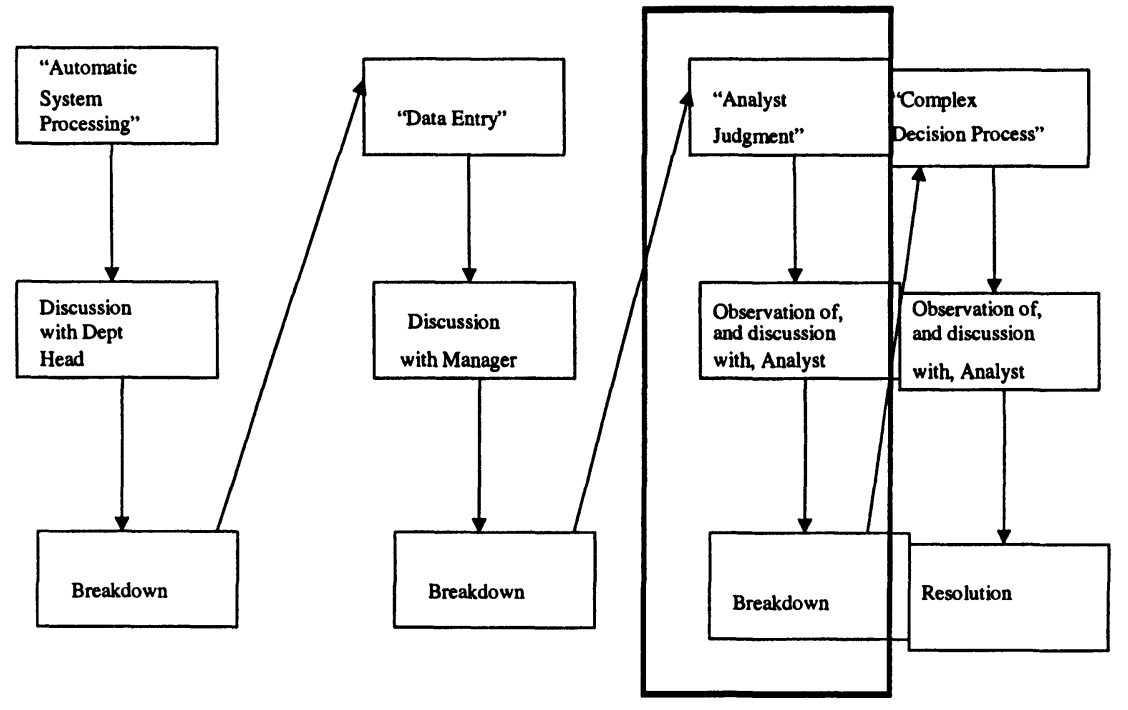

Figure 8: Breakdown of Schema 3:

Evaluate Holdings As “Analyst's Judgment” 


\subsection{Schema 4: Evaluating Holdings is a "Complex Decision Process"}

Evaluate Holdings

Aualyst: Hayes

Client: Mens Wearhouse, Inc.

Investment Manager: Crainer, Rosenthal \& McGlyun, Inc.

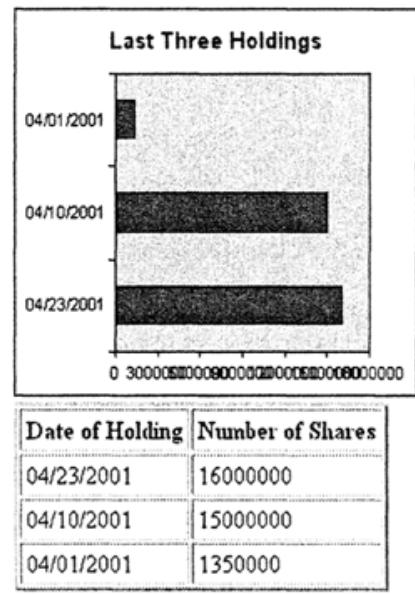

\begin{tabular}{|c|c|}
\hline Account Aggregration & 15855000 \\
\hline From Schedule 13F & 13500000 \\
\hline $\begin{array}{l}\text { Last Holding: } \\
\text { Holding Date: } \\
\text { Confidence Level } \\
\text { Comments: }\end{array}$ & $\begin{array}{l}14000000 \\
05 / 01 / 2001 \\
75 \% \\
\text { Account Asgregat }\end{array}$ \\
\hline $\begin{array}{l}\text { Investment Manager Contact: } \\
\text { Position: } \\
\text { Telephone Number: }\end{array}$ & $\begin{array}{l}\text { Joe Smith } \\
\text { Manager } \\
212-346-1000\end{array}$ \\
\hline \multicolumn{2}{|l|}{ Your Judgement: } \\
\hline \multicolumn{2}{|l|}{ Confidence Level: } \\
\hline \multicolumn{2}{|l|}{ Notes: } \\
\hline Submit New Holding & Reset New Holding \\
\hline $\begin{array}{l}\text { If you want to view the holdings } \\
\text { of Investment Managers using sin } \\
\text { investment technique, click here. }\end{array}$ & \\
\hline
\end{tabular}

Figure 9: Schema 4: Evaluate Holdings as "A Complex Decision Process"

The analyst who worked on the prototype mentioned that two of the "missing" factors in the prototype were a "historical trend analysis" of whether an investment manager was buying or selling a client's stock and a "similar investors' trend analysis" (i.e., were other investment managers using similar investment strategies, such as a growth-oriented or a conservative investment strategy, buying or selling that client's stock?). So, my next revision of my schema (Schema 4) was that "evaluating holdings is a complex decision process" involving several types of "trend analysis" (as represented in Figure 9 and Figure 10). Figure 10 represents the buying or selling trends of "like-minded" investors presented in a new window that opens up from the "main window" represented in Figure 9. 


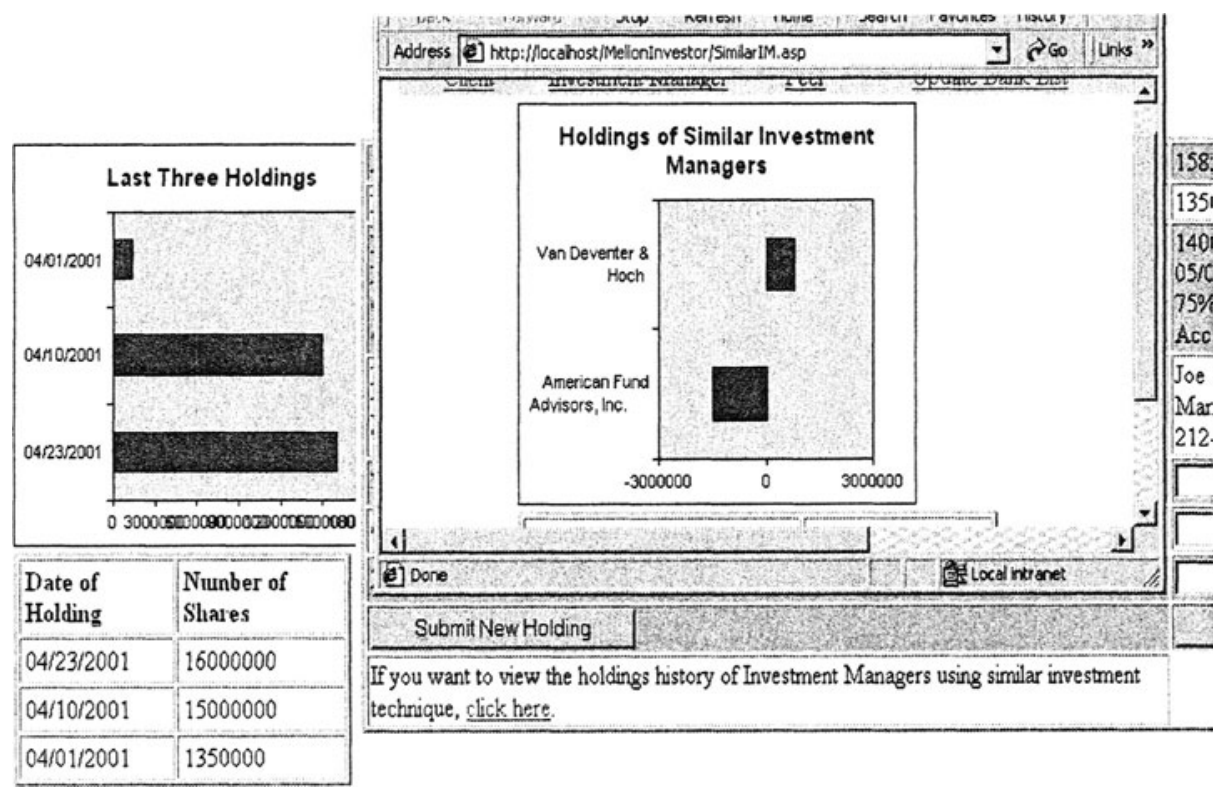

Figure 10: Schema 4 (continued)

\section{COMPARISON OF THE STRIP RESOLUTION PROCESS WITH OTHER APPROACHES FOR DESIGNING AND DEVELOPING WEB APPLICATIONS:}

UML-based approaches are among the most widely prescribed approaches for web application design and development (Conallen, 2000; Reed, 2002, Rosenberg and Scott, 2001). These approaches suggest an iterative process involving three types of UML diagrams, Use Cases, Class Diagrams, and Sequence Diagrams. They suggest Use Cases for determining requirements, Class Diagrams for representing the static structure of the application, and Sequence Diagrams for representing the dynamic aspects of the application.

Another set of approaches that are prescribed for designing and developing web applications fall under the category of Agile Methodologies. Two among these agile development methodologies are Extreme Programming (Beck, 2000) and Adaptive Software Development (Highsmith, 2000; Cockburn, 2002). These agile methodologies accept that requirements will change. In fact, they embrace this change by emphasizing 
an iterative process of analysis, design, and development, by having very short release cycles, by maintaining close communication between developers and customers, and by keeping the design as simple as possible.

My use of the Strip Resolution process complements these UML-based and Agile development approaches. These development approaches have attempted to close the technical gap between the user and the technology. However, there is still an underlying reliance on process, notation, and tools. Since the Strip Resolution process is an ethnographic research approach based on anthropological traditions, its emphasis is on understanding the actors and their behaviors in their native environment. So, in contrast to the other development approaches, the Strip Resolution process attempts to close the "understanding gap" between developers and users. A well-known Agile development methodologist, Alistair Cockburn, lends credence to the approach I am adopting by explicitly stating the need for methodologists to team up with ethnographers, sociologists, and anthropologists:

"We are still in the infancy of naming what is really happening on software development projects. The answer is not process, modeling, or mathematics, although those play parts. The answer has much more to do with craft, community, pride, and learning [...]. The next step is for methodologists to partner with ethnographers, sociologists, and anthropologists to see if they have words to capture other parts of the [software development] experience" (p.6, Cockburn, 2002).

The following is an example of how the Strip Resolution process could complement the UML-based development methodologies. If, in my role as a developer, I had used a UML Use Case to represent my initial understanding, I would not have identified anything other than one Use Case for "Evaluate Holdings" (without identifying the Use Cases it "uses."). Also, I would have identified this use case as "system generated" and not associated with a stock analyst actor as shown in Figure 11. I probably would have been able to refine the Use Case to better reflect the functional requirements in subsequent iterations. However, I would not have been able to understand and represent all the associated use cases (represented in Figure 12) without bridging the "understanding gap" between myself in the role of a developer and the user. The Strip Resolution process provides this bridge across the "understanding gap." Also, the strip resolution process provides a means of checking the validity of each Use Case by applying each Use Case "schema" on several "strips" derived from the users' environment. The validity of each Use Case would depend on whether that Use Case makes the strip understandable. 


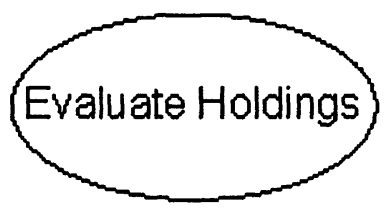

Figure 11: Use Case Diagram: Before use of strip resolution process

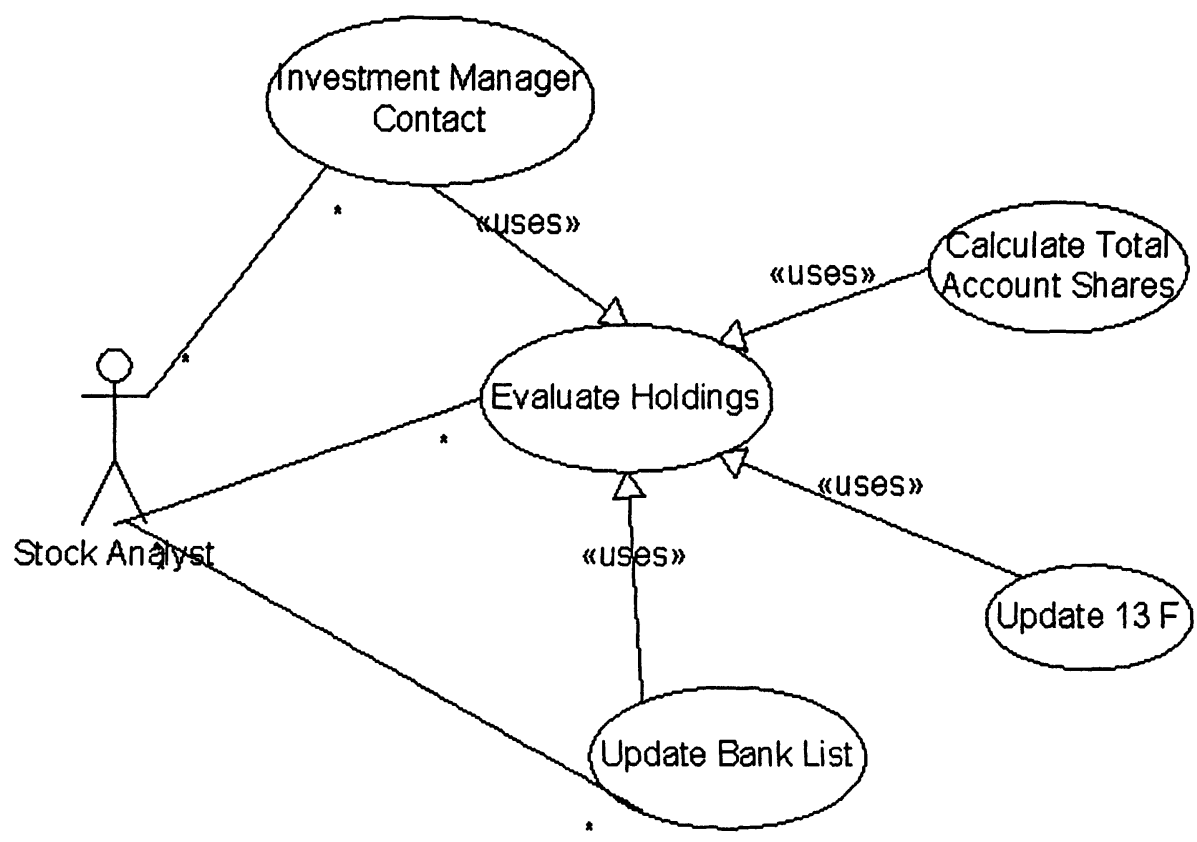

Figure 12: Use Case Diagram: Complemented by Strip Resolution process

\section{CONCLUSION}

The strip resolution process has mainly been used in sociological and anthropological studies as a "researcher's tool" to better understand people from societies different from the researchers'. However, as shown in my use of the strip resolution process (represented in summary form in Figure 13) to understand what it means for a stock analyst to "evaluate stock holdings," this process can not only be used as a researcher's tool but as an effective tool (by itself or as a complement to other development methodologies such 
as the UML-based methodologies discussed in Section 4) to understand work processes and user requirements to better design and develop applications.

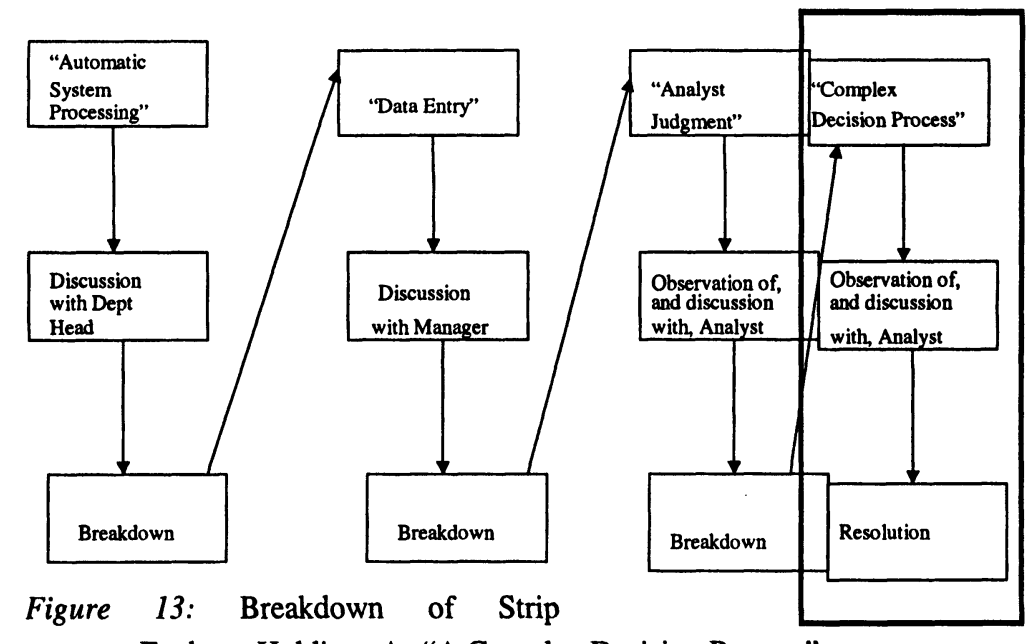

Evaluate Holdings As "A Complex Decision Process"

\section{REFERENCES}

1. Agar, M. (1986) Speaking Of Ethnography. Sage Publications, Beverly Hills, CA.

2. Baskerville, R. (1999) "Investigating Information Systems With Action Research," Communications of the Association for Information System, Volume 2, Article 19, October 1999.

3. Beck, K. (2000) Extreme Programming Explained: Embrace Change. Addison-Wesley, New York, NY.

4. Cockburn, A. (2002) Agile Software Development. Addison-Wesley, New York, NY.

5. Highsmith, J. and Orr, K. (2000) Adaptive Software Development: A Collaborative Approach to Managing Complex Systems, Dorset House

6. Reed, P. (2002) Developing Application with JAVA and UML. AddisonWesley, New York, NY.

7. Rosenberg, R. and Scott, K. (2001) Applying Use Case Driven Object Modeling With UML: An Annotated E-Commerce Example. Addison-Wesley, New York, NY.

8. Whyte, W. (1989) "Introduction," in Whyte, W (editor) Action Research For The Twenty-First Century: Participation, Reflection, and Practice, a special issue of American Behavioral Scientist, Volume 32, Number 5, May/June 1989.

9. Whyte, W., Greenwood, D., Lazes, P. (1989) "Participatory Action Research" in Whyte, W (editor) Action Research For The Twenty-First Century: Participation, Reflection, and Practice, a special issue of American Behavioral Scientist, Volume 32, Number 5 , May/June 1989. 